\title{
LAS-CDMA USING VARIOUS TIME DOMAIN CHIP-WAVEFORMS
}

\author{
H. Wei and L. Hanzo \\ School of ECS, University of Southampton, SO17 1BJ, UK. \\ Tel: +44-23-8059-3125, Fax: +44-23-8059-4508 \\ Email: $1 h^{1} @ e c s . s o t o n . a c . u k, ~ h t t p: / /$ www-mobile.ecs.soton.ac.uk
}

\begin{abstract}
LAS CDMA exhibits a significantly better performance than that of classic random code based DS-CDMA, when operating in a quasi-synchronous scenario. Classic frequency-domain raised cosine Nyquist filtering is known to show the best possible performance, but its complexity may be excessive in highchip-rate systems. Hence in these systems often low-complexity time-domain waveform shaping is considered. Motivated by this fact, the achievable performance of LAS-CDMA is investigated in conjunction with three different time-limited chipwaveforms, which exhibit an infinite bandwidth. The raised cosine time-domain waveform based DS-CDMA system is shown to achieve the best performance in the context of a strictly band-limited system, because its frequency-domain spectral side-lobes are relatively low.
\end{abstract}

\section{INTRODUCTION}

In Direct Sequence Code Division Multiple Access (DS-CDMA) systems, the spreading sequences characterize the associated Inter Symbol Interference (ISI) as well as the Multiple Access Interference (MAI) properties [1]. Traditional spreading sequences, such as $m$-sequences [1], Gold codes [1] and Kasami codes [1] exhibit non-zero off-peak auto-correlations and cross-correlations, which results in a high MAI in case of asynchronous uplink transmissions. A family of orthogonal codes is constituted by Walsh codes [1] and orthogonal Gold codes, which do retain their orthogonality in case of perfect synchronization, but they also exhibit non-zero off-peak auto-correlations and cross-correlations in asynchronous scenarios. Consequently, these correlation properties limit the achievable performance in asynchronous scenarios. Hence traditional DS-CDMA cellular systems are interference limited and suffer from the so-called 'near-far' effects, unless complex interference cancellers [1] or multi-user detectors [1] are employed for combating these adverse effects. This results in costly and 'power-hungry' implementations. All these limitations are imposed by the imperfect correlation properties of the spreading sequences employed. Hence, considerable research efforts have been invested in designing spreading sequences, which exhibit zero correlation values, when the relative delay-induced code offset is in the so-called Zero Correlation Zone (ZCZ) or Interference Free Window (IFW) of the spreading code. The attractive family of Large Area Synchronized (LAS) CDMA spreading sequences is constituted by the combination of the so-called Large Area (LA) codes [2,3] and Loosely Synchronous (LS) codes [4]. The resultant LAS codes exhibit an IFW, where the off-peak aperiodic autocorrelation values as well as the aperiodic cross-correlation values become zero, resulting in zero ISI and zero MAI, provided that the time-offset of the codes is within the IFW. Figure 1 characterizes the correlation properties of a traditional random spread- ing code and those of an $\operatorname{LS}(4,8,8)[3]$ code. Li [2,5] proposed the employment of the so-called LAS codes, which exhibit zero auto-correlation and cross-correlation in a limited-offset range of $[-\iota, \iota]$-chips. In $[6,7]$ the authors of this contribution investigated

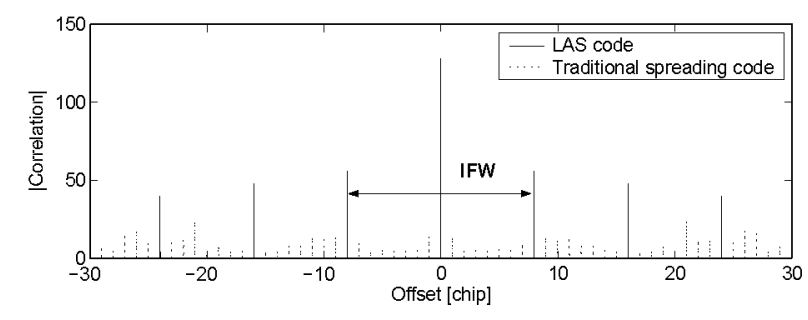

Figure 1: Correlation of both LAS and random spreading sequences

the performance of LAS-CDMA, which exhibited a significantly better performance than that of classic CDMA, when operating in a quasi-synchronous scenario.

In this paper we investigated the performance of LAS CDMA in conjunction with various chip-waveforms in the context of both an infinite bandwidth and a strictly bandlimited scenario. More explicitly, the different chip-waveform designs are expected to incur different amounts of multiple access interference (MAI) as well as requiring different signalling bandwidths. Hence we investigated a LAS-CDMA system in conjunction with both rectangular chipwaveforms as well as half-sine and raised-cosine chip-waveforms. Closed-form formulas are derived for evaluating the performance of LAS CDMA, when invoking different chip-waveforms.

The organization of this paper is as follows. Section 2 describes our system model, while Section 3 characterizes the achievable analytical BER performance of LAS-CDMA when using various chips waveforms. Section 4 introduces the time-limited chipwaveforms studied, followed by our numerical performance study in Section 5. Finally, Section 6 offers our conclusions.

\section{SYSTEM MODEL}

Let us consider an asynchronous $K$-user DS-CDMA communication system, where each user is assigned a unique signature waveform $\mathbf{c}_{k}(t)=\sum_{i=0}^{G-1} c_{k i} \psi_{T_{c}}\left(t-i T_{c}\right)$. The chip-sequence $c_{k i} \epsilon$ $\frac{1}{\sqrt{G}}\{+1,-1,0\}$ represents the spreading sequence of the $k$ th user and in this contribution we will consider PN random codes as well as the LAS spreading codes $[2,3]$. The processing gain is denoted by $G$, which obeys $G=T_{s} / T_{c}$, and $\psi_{T_{c}}(t)$ represents the chip-waveform having an energy of $\int_{0}^{T_{c}} \psi_{T_{c}}^{2}(t) d t=T_{c}$. Again, 
we considered three chip-waveforms, namely rectangular, raisedcosine and half-sine chip-waveforms. For convenience, we define the normalized chip-waveform as $\hat{\psi}(t)=\psi_{T_{c}}\left(t / T_{c}\right)$. For the band-limited system considered, the normalized chip-waveform $\hat{\psi}(t)$ satisfies:

$$
\int_{-\infty}^{+\infty} \hat{\psi}\left(t-n_{1}\right) \hat{\psi}^{*}\left(t-n_{2}\right) d t=\delta\left(n_{1}, n_{2}\right)
$$

where $\delta\left(n_{1}, n_{2}\right)=1$ for $n_{1}=n_{2}$, and 0 for $n_{1} \neq n_{2}$. From Eq.(1), we have:

$$
\sum_{n=-\infty}^{\infty} \hat{\psi}^{2}(n)=1
$$

Consequently, when the $K$ users' signals are transmitted over a frequency-selective fading channel, the received complex-valued low-pass equivalent signal at a given base station can be expressed as:

$$
\begin{aligned}
R(t)= & \sum_{k=1}^{K} \sum_{l=0}^{L_{p}-1} \sqrt{2 P_{k}} \mathbf{c}_{k}\left(t-l T_{c}-\tau_{k}\right) \\
& b_{k}\left(t-l T_{c}-\tau_{k}\right) h_{k l} \exp \left(j \theta_{k l}\right)+N(t),
\end{aligned}
$$

where $N(t)$ is the complex-valued low-pass-equivalent AWGN having a double-sided spectral density of $N_{0}$ and $\tau_{k}$ is the propagation delay of user $k$, while $L_{p}$ is the total number of resolvable paths. Furthermore, the complex low-pass equivalent representation of the Channel Impulse Response (CIR) encountered by the $k$ th user is given by [8]

$$
h_{k}(t)=\sum_{l=0}^{L_{p}-1} h_{k l} \delta\left(t-l T_{c}\right) \exp \left(j \theta_{k l}\right),
$$

where $h_{k l}$ represents the Nakagami-distributed fading envelope, $l T_{c}$ is the relative delay of the $l$ th path of user $k$ with respect to the main path, while $L_{p}$ is the total number of resolvable multipath components. Furthermore, $\theta_{k l}$ is the uniformly distributed phaseshift of the $l$ th multipath component of the channel and $\delta(t)$ is the Kronecker Delta-function. More explicitly, the $L$ multipath attenuations $\left\{h_{k l}\right\}$ are independent Nakagami distributed random variables having a Probability Density Function (PDF) of [9-11]:

$$
\begin{aligned}
p\left(h_{k l}\right) & =M\left(h_{k l}, m_{k l}, \Omega_{k l}\right), \\
M(R, m, \Omega) & =\frac{2 m^{m} R^{2 m-1}}{\Gamma(m) \Omega^{m}} e^{(-m / \Omega) R^{2}},
\end{aligned}
$$

where $\Gamma(\cdot)$ is the gamma function [8], and $m_{k l}$ is the Nakagami$m$ fading parameter, which characterizes the severity of the fading for the $l$-th resolvable path of user $k$ [12] . Specifically, $m_{k l}=1$ represents Rayleigh fading, $m_{k l} \rightarrow \infty$ corresponds to the conventional Gaussian scenario and $m_{k l}=1 / 2$ describes the so-called one-sided Gaussian fading, i.e. the worst-case fading condition. The Rician and log-normal distributions can also be closely approximated by the Nakagami distribution in conjunction with values of $m_{k l}>1$. The parameter $\Omega_{k l}$ in Equation 5 is the second moment of $h_{k l}$, i.e. we have $\Omega_{k l}=E\left[\left(h_{k l}\right)^{2}\right]$. We assume a negative exponentially decaying Multipath Intensity Profile (MIP) given by $\Omega_{k l}=\Omega_{k 0} e^{-\eta l}, \eta \geq 0, l=0, \ldots, L_{p}-1$, where $\Omega_{k l}$ is the average signal strength corresponding to the first resolvable path and $\eta$ is the rate of average power decay.

\section{BER ANALYSIS}

Let the first user be the user-of-interest and consider a receiver using de-spreading as well as multipath diversity combining. The conventional matched filter based RAKE receiver using MRC may be invoked for detection, where we assume that the RAKE receiver is capable of combining $L_{r}$ number of diversity paths.

Let us assume that we have achieved time synchronization and perfect estimates of the channel magnitudes and phases are available. The individual matched filter outputs are appropriately delayed, in order to coherently combine the $L_{r}$ number of path signals processed by the RAKE combiner. The $l$ th RAKE combiner finger's output $Z_{k l}$ is sampled at $t=T+l T_{c}+\tau_{k}$, in order to detect the $k$ th user's transmitted symbol $b_{k}[0]$, which is expressed as:

$$
Z_{k l}=D_{k l}+I_{k l}
$$

where $D_{k l}$ represents the desired direct Line-of-Sight (LOS) component, which can be expressed as:

$$
D_{k l}=\sqrt{2 P} T_{s} b_{k}[0] h_{k l}^{2} .
$$

In Eq.(7) $b_{k}[0]$ is the first bit transmitted by the $k$ th BPSK user and we have $b_{k}[0] \in\{+1,-1\}$. Hence, the interference plus noise term $I_{k}$ in Eq.(6) may be expressed as:

$$
I_{k l}=I_{k l}[S]+I_{k l}[M]+N_{k l}
$$

where $I_{k l}[S]$ represents the multipath interference imposed by the user-of-interest. Explicitly, $I_{k l}[S]$ may be expressed as:

$$
I_{k l}[S]=\sqrt{2 P} T_{s} h_{k l} \sum_{\substack{l_{p}=0 \\ l_{p} \neq l}}^{L_{p}-1} h_{k l_{p}} \exp \left(j \theta_{k l_{p}}\right)\left\{\rho_{k k} \hat{\psi}(0)\right\}(9)
$$

where the term $\rho_{k k}(n)$ is formulated as:

$$
\rho_{k k}=\frac{1}{G} \sum_{i=0}^{G-1} c_{k}[i]^{*} c_{k}\left[i-l_{p}\right]
$$

Furthermore, $I_{k l}[M]$ of Eq.(8) represents the multiuser interference inflicted by the $K-1$ interfering users, which is expressed as:

$$
\begin{aligned}
I_{k l}[M]= & \sqrt{2 P} T_{s} h_{k l} \sum_{\substack{k^{\prime}=1 \\
k^{\prime} \neq k}}^{K} \sum_{l_{p}=0}^{L_{p}-1} h_{k^{\prime} l_{p}} \exp \left(j \theta_{k l_{p}}\right) \\
& \times\left\{\rho_{k k^{\prime}}(l) \hat{\psi}\left(\tau_{k}\right)+\rho_{k k^{\prime}}(r) \hat{\psi}\left(1-\tau_{k}\right)\right\},
\end{aligned}
$$

and we have:

$$
\begin{aligned}
\rho_{k k^{\prime}}(l) & =\frac{1}{G} \sum_{i=0}^{G-1} c_{k}[i]^{*} c_{k^{\prime}}\left[i-l_{p}-1\right] . \\
\rho_{k k^{\prime}}(r) & =\frac{1}{G} \sum_{i=0}^{G-1} c_{k}[i]^{*} c_{k^{\prime}}\left[i-l_{p}\right] .
\end{aligned}
$$

It was shown in $[13,14]$ for a random PN spreading sequence that the random variables $\rho_{k k}, \rho_{k k^{\prime}}(l)$ and $\rho_{k k^{\prime}}(r)$ may be modelled as complex Gaussian random variables having a mean of zero and a variance of $1 / G$. Therefore, the variance of the term $I_{k l}[S]$ of 
Eq.(8), which was explicitly formulated in Eq.(9) can be expressed as:

$$
\operatorname{Var}\left\{I_{k l}[S]\right\}=2 P T_{s}^{2} h_{k l}^{2} \frac{1}{G} \Omega_{0}\left[q\left(L_{p}, \eta\right)-1\right]
$$

Similarly, the variance of the term $I_{k l}[M]$ formulated in Eq.(11) can be expressed as:

$$
\begin{aligned}
\operatorname{Var}\left\{I_{k l}[M]\right\}= & 2 P T_{s}^{2} h_{k l}^{2} \Omega_{0} K q\left(L_{p}, \eta\right) \frac{1}{G} \\
& E\left[\hat{\psi}^{2}(\tau)+\hat{\psi}^{2}(1-\tau)\right] \\
= & 2 P T_{s}^{2} h_{k l}^{2} \Omega_{0} K q\left(L_{p}, \eta\right) \frac{1}{G} E[\phi(\tau)],
\end{aligned}
$$

where $E[\phi(\tau)]=E\left[\hat{\psi}^{2}(\tau)+\hat{\psi}^{2}(1-\tau)\right]$ defines the interference factor ${ }^{1}$ associated with a specific chip pulse shape, which predetermines the amount of the MAI imposed by different chipwaveforms. The interference factor associated with a specific pulse shape is expected to decrease as the excess bandwidth increases. In other words, we are capable of reducing the MAI imposed on other users at the cost of decreasing the attainable spectral efficiency, as it will be demonstrated and it is possible to strike a trade-off between the processing gain $G$ and the excess bandwidth. Furthermore, $\tau$ in Equation 15 is a random variable uniformly distributed in $[0,1]$, and $E[\phi(\tau)]$ can be expressed as $E[\phi(\tau)]=2 E\left[\hat{\psi}^{2}(\tau)\right]$.

Finally, the noise term of Eq.(8) can be expressed as:

$$
N_{k l}=h_{k l} \int_{0}^{T_{s}} n(t) c[t] \cos \left(2 \pi f_{c} t+\theta_{k l}\right) d t
$$

which is a Gaussian random variable having zero mean and a variance of $N_{0} T_{s} h_{k l}^{2}$.

In $[6,7]$ the authors of this contribution introduced the MPI and MAI interference reduction factors of $\Upsilon_{S}(l)$ and $\Upsilon_{M}(l)$ for LAS codes, which reflected the corresponding MAI and MPI reduction achievable in comparison to random codes. Hence, the corresponding MAI and MPI variance for LAS codes can be expressed as:

$$
\begin{aligned}
\operatorname{Var}\left\{I_{k l}[S]\right\} & =2 P T_{s}^{2} h_{k l}^{2} \frac{1}{G} \Omega_{0} \Upsilon_{S}(l) \\
\operatorname{Var}\left\{I_{k l}[M]\right\} & =2 P T_{s}^{2} h_{k l}^{2} \Omega_{0} K \frac{1}{G} \Upsilon_{M}(l) E[\phi(\tau)]
\end{aligned}
$$

The MRC's decision variable $Z_{k}$, is constituted by the sum of the RAKE fingers' output, which can be expressed as:

$$
Z_{k}=\sum_{l=0}^{L_{r}-1} Z_{k l}
$$

Following a similar procedure to that described in $[6,7]$ and a range of arduous manipulations, the corresponding bit error ratio (BER) can be expressed as:

$$
\begin{aligned}
P_{b}(E) & =Q\left(\sqrt{\frac{\left(\mathrm{E}\left[Z_{k}\right]\right)^{2}}{\operatorname{Var}\left[Z_{k}\right]}}\right) \\
& =\frac{1}{\pi} \int_{0}^{\pi / 2} \prod_{l=0}^{L_{r}-1}\left(\frac{m \sin ^{2} \theta}{\bar{\gamma}_{l}+m \sin ^{2} \theta}\right)^{m} d \theta,
\end{aligned}
$$

\footnotetext{
${ }^{l}$ Since different chip-waveforms have different bandwidth requirements and hence inflict different amounts of MAI, the different chipwaveforms are characterized by their interference factors.
}

where we have $\bar{\gamma}_{l}=\gamma_{c} e^{-\eta l} l=0,1, \ldots, L_{r}-1$, and $L_{r}$ represents the number of RAKE receiver fingers, while $\eta$ is the negative exponential Channel Impulse Response (CIR) tap decay factor. For the random codes, the corresponding $\gamma_{c}$ is given by:

$\gamma_{c}=\left[\frac{K q\left(L_{p}, \eta\right) E[\phi(\tau)]}{G}+\frac{q\left(L_{p}, \eta\right)-1}{G}+\left(\frac{\Omega E_{b}}{N_{0}}\right)^{-1}\right]^{-1}$,

while for LAS CDMA we have

$$
\gamma_{c}=\left[\frac{\Upsilon_{S}(l)}{G}+\frac{K \Upsilon_{M}(l) E[\phi(\tau)]}{G}+\left(\frac{\Omega_{0} E_{b}}{N_{0}}\right)^{-1}\right]^{-1} .
$$

\section{CHIP WAVEFORMS}

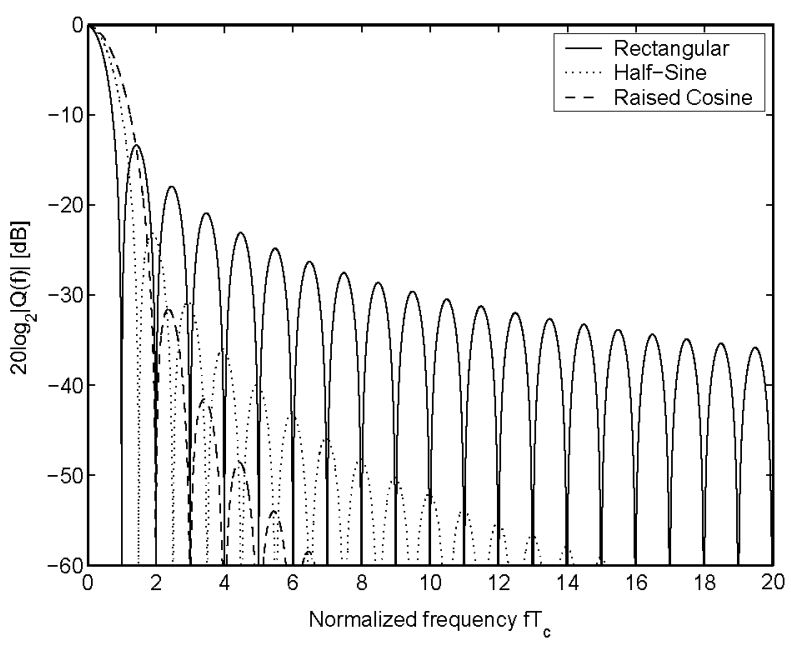

Figure 2: The logarithmic power density spectrum $|Q(f)|^{2}$ of the rectangular, half-sine and raised cosine time-domain chipwaveforms.

For time-limited chip-waveforms, the corresponding frequencydomain spectral density function $Q(f)$ may be expressed with the aid of the Fourier transform $Q(f)=\mathcal{F}\left\{\psi_{T_{c}}(t)\right\}$. Figure 2 portrays the corresponding power spectral density function $|Q(f)|^{2}$. We considered the fractional energy containment bandwidth definitions of $[15,16]$ for the sake of characterizing these time-limited waveforms. The stringent definition of $\frac{\int_{-W}^{W} Q^{2}(f) d f}{\int_{-\infty}^{\infty} Q^{2}(f) d f} \geq 99.995 \%$ was used for quantifying the required bandwidth as the band containing $99.995 \%$ of the chip-waveform's power in the context of the finite time duration signalling pulses considered. By contrast, in terms of the Nyquist excess bandwidth $\beta$, which quantifies the required bandwidth, we have:

$$
W=\frac{1+\beta}{2 T_{c}} .
$$

In [14,17], Dallas and Pavlidou investigated the interference factor, $E[\phi(\beta, \tau)]$ defined in the context of Eq.(15), which was found to be $0.666,0.596$ and 0.582 for the rectangular, half-sine and raised cosine chip-waveforms, respectively. Therefore, following Eq.(24) and the above definition of the energy containment bandwidth, the required excess bandwidths are summarized in Table 1 for both of 
the above mentioned energy containment factors, along with the corresponding interference factors for the three time-limited chipwaveforms considered.

\begin{tabular}{|l|l|l|}
\hline Waveform & $\begin{array}{l}\text { Energy containment } \\
\text { bandwidth } \geq 99.995 \%\end{array}$ & $\begin{array}{l}\text { Interference } \\
\text { factor } E[\phi(\tau)]\end{array}$ \\
\hline Rectangular & $\beta=38.34$ & 0.666 \\
\hline Half-Sine & $\beta=12.90$ & 0.596 \\
\hline Raised Cosine & $\beta=5.58$ & 0.482 \\
\hline
\end{tabular}

Table 1: The required excess bandwidth $\beta$ and the corresponding interference factors $E[\phi(\tau)]$ for various time-domain chip-pulse shapes.

\section{NUMERICAL RESULTS}

In our investigations we compared a traditional random code and a LAS-code based CDMA system, both of which have the same chip-rate in conjunction with various chip-waveforms. Again, LAS codes exhibit a zero-valued IFW, which does not contribute towards the spreadign gain achievable in a given total bandwidth. Hence the corresponding spreading gain remains slightly lower than that of traditional random codes having the same number of chips per spreading sequence. This was duly taken into account in our study for the sake of a fair comparison. When using the parameters of the LAS-2000 [18] system, the relationship of the corresponding spreading gains satisfied $G=G_{R A N}=1.17 G_{L A S}$, where $G_{R A N}$ and $G_{L A S}$ are the effective spreading gains of the random codes and LAS codes, respectively. For simplicity's sake, we assume that all paths have the same Nakagami fading parameter, i.e. we have $m_{l}=m, l=0, \ldots, L_{r}-1$. Furthermore, we assume that both the random and LAS-code based systems have a chip rate of $1.2288 \mathrm{M}$ chips and the channel's dispersive delay spread is $\tau_{c h}=3 \mu s$. Hence the number of resolvable paths is $L_{p}=\left\lfloor\frac{\tau_{c h}}{T_{c}}\right\rfloor+1=4$. The width of the IFW was $\iota=3 T_{c}$ and the maximum asynchronous delay difference of the uplink mobile transmitters was $\tau_{\max }=2 T_{c}$, while the negative exponential MIP decay factor was $\eta=0.2$.

Figure 3 portrays the BER versus channel SNR performance comparison of random code based classic CDMA and LAS CDMA in conjunction with the various chip-waveforms considered, when communicating over an infinite bandwidth Nakagami- $m$ channel having $m=1$, i.e. when the excess bandwidth was $\beta \rightarrow \infty$, and $K=32$ users were supported. From this figure we may observe that the LAS-CDMA system exhibited a significantly better BER performance than the traditional DS-CDMA system in the quasi-synchronous scenarios considered. Furthermore, the different interference factors of the different chip-waveforms had a relatively modest effect on the associated MAI of both the random code based and on the LAS-CDMA systems. The raised-cosine chip-waveform inflicted the lowest amount of MAI.

By contrast, Figure 4 characterizes the BER versus channel SNR performance of both the random code based classic CDMA and LAS CDMA in conjunction with various chip-waveforms, when communicating over a band-limited Nakagami- $m$ channel having $m=1$. The energy containment requirement was again

$$
\frac{\int_{-W}^{W} Q^{2}(f) d f}{\int_{-\infty}^{\infty} Q^{2}(f) d f} \geq 99.995 \%
$$

, resulting in the excess bandwidth values listed in Table 1 . The total bandwidth of all the waveforms was set to $G(1+\beta)=$

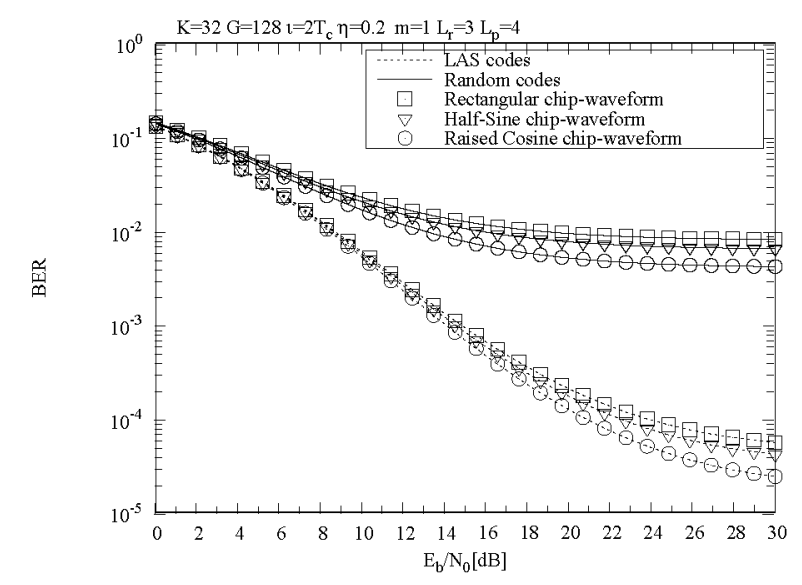

Figure 3: BER versus channel SNR performance comparison of random code based classic CDMA and LAS CDMA using three different chip-waveforms when communicating over a Nakagami$m$ channel having $m=1$, when the excess bandwidth obeys $\beta \rightarrow$ $\infty$.

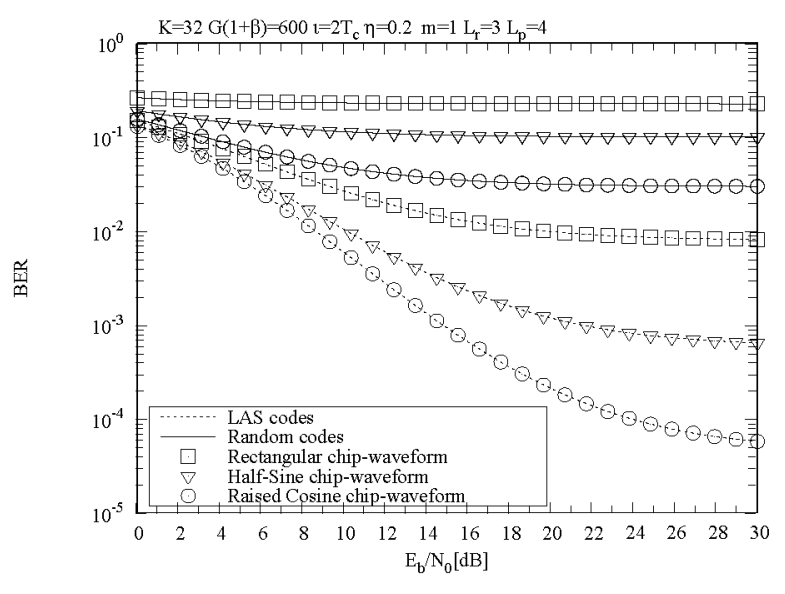

Figure 4: BER versus channel SNR performance comparison of random code based classic CDMA and LAS CDMA using three different chip-waveforms, when communicating over a Nakagami$m$ channel having $m=1$. The energy containment requirement was satisfied according to the strictly bandlimited condition [15, $16]$ of $99.995 \%$ and the total bandwidth was fixed to $G(1+\beta)=$ 600 , while $K=32$ users were supported. 


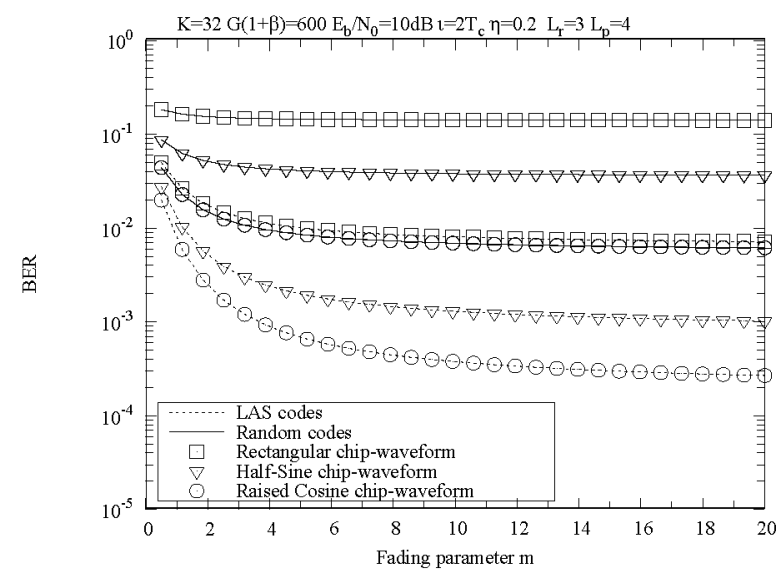

Figure 5: BER versus Nakagami fading parameter, $m$, comparison of random code based classic CDMA and LAS CDMA, when communicating over different fading channels associated with different Nakagami fading parameters $m$. The energy containment requirement was satisfied according to the strictly bandlimited condition $[15,16]$ of $99.995 \%$ and the total bandwidth was $G(1+\beta)=600$.

600 and $K=32$ users were supported. We observe in Figure 4 that the raised-cosine chip-waveform exhibited a significantly better performance than that of the rectangular and half-sine chipwaveforms.

Figure 5 shows the performance of these two systems in conjunction with various chip-waveforms, when communicating over different strictly bandlimited fading channels associated with different Nakagami fading parameters $m$. Finally, Figure 6 portrays the BER versus number of users comparison of both random code based classic CDMA and LAS CDMA, when communicating over a strictly bandlimited Nakagami- $m$ channel having $m=1$ at a bit energy to noise ration of $E_{b} / N_{0}=30 \mathrm{~dB}$.

\section{CONCLUSIONS}

In this paper we have investigated the achievable performance of LAS-CDMA in conjunction with three different time-limited chipwaveforms. The raised cosine time-domain waveform based DSCDMA system achieved the best performance in the context of a strictly band-limited system, because its frequency-domain spectral side-lobes are relatively low. LAS CDMA exhibited a significantly better performance than that of classic random code based DS-CDMA, when operating in a quasi-synchronous scenario.

\section{REFERENCES}

[1] L. Hanzo, L. L. Yang, E. L. Kuan, and K. Yen, Single- and MultiCarrier DS-CDMA. John Wiley and IEEE Press, 2003, 1060 pages.

[2] D. Li, "A High Spectrum Efficient Multiple Access Code," Chinese Journal of Electronics, vol. 8, pp. 221-226, July 1999.

[3] B. J. Choi and L. Hanzo, "On the Design of LAS Spreading Codes," in IEEE VTC 2002 Fall Conference, (Vancouver, Canada), pp. 21722176, September 2002.

[4] S. Stańczak, H. Boche, and M. Haardt, "Are LAS-codes a Miracle?," in GLOBECOM '01, vol. 1, (San Antonio, Texas), pp. 589593, November 2001.

[5] D. Li, "The Perspectives of Large Area Synchronous CDMA Technology for the Fourth-Generation Mobile Radio," IEEE Communications Magazine, vol. 41, pp. 114 - 118, March 2003.

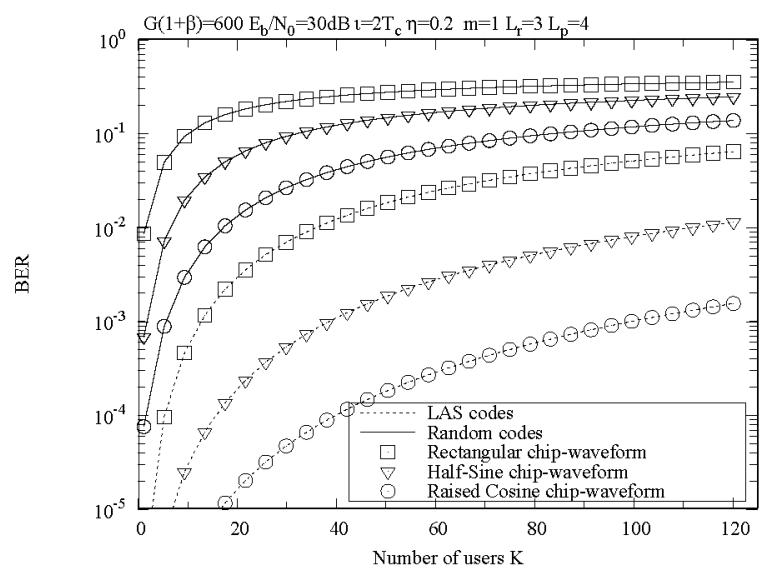

Figure 6: BER versus the number of user comparison of random code based classic CDMA and LAS CDMA, when communicating over a Nakagami- $m$ channel having $m=1$. The energy containment requirement was satisfied according to the strictly bandlimited condition $[15,16]$ of $99.995 \%$ and the total bandwidth was $G(1+\beta)=600$.

[6] H. Wei and L. Hanzo, "On the Performance of LAS-CDMA," To appear in IEEE Transactions on Wireless Communication.

[7] H. Wei and L. Hanzo, "On the uplink performance of LAS-CDMA," In Proceedings of VTC'05 Spring, Stockholm, May 2005, CD ROM, 2005.

[8] J. G. Proakis, Digital Communications. Mc-Graw Hill International Editions, 3rd ed., 1995.

[9] N. Nakagami, "The $m$-Distribution, a General Formula for Intensity Distribution of Rapid Fading," in Statistical Methods in Radio Wave Propagation (W. G. Hoffman, ed.), Oxford, England: Pergamon, 1960.

[10] T. Eng and L. B. Milstein, "Coherent DS-CDMA Performance in Nakagami Multipath Fading," IEEE Transactions on Communications, vol. 43, pp. 1134-1143, February/March/April 1995.

[11] V. Aalo, O. Ugweje, and R. Sudhakar, "Performance Analysis of a DS/CDMA System with Noncoherent $M$-ary Orthogonal Modulation in Nakagami Fading," IEEE Transactions on Vehicular Technology, vol. 47, pp. 20-29, February 1998.

[12] M.-S. Alouini and A. J. Goldsmith, "A Unified Approach for Calculating Error Rates of Linearly Modulated Signals over Generalized Fading Channels," IEEE Transactions on Communications, vol. 47, pp. 1324-1334, September 1999.

[13] J. H. Cho and J. S. Lehnert, "Performance of a Spatio-Temporal Matched Filter for DS/SSMA Communications with Random Quadriphase Spreading Sequences," IEEE Journal on Selected Areas in Communications, vol. 18, pp. 1505-1515, May 2000.

[14] J. H. Cho and J. S. Lehnert, "An Optimal Signal Design for BandLimited Asynchronous DS-CDMA Communications," IEEE Transactions on Information Theory, vol. 48, pp. 1172-1185, May 2002.

[15] F. Amoroso, "The Bandwidth of Digital Data Signals," IEEE Communications Magazine, vol. 18, pp. 13-24, November 1980.

[16] Y. C. Yoon, "A Simple and Accurate Method of Probability of Bit Error Analysis for Asynchronous Band-Limited DS-CDMA Systems," IEEE Transactions on Communications, vol. 50, pp. 656-663, April 2002.

[17] P. I. Dallas and F.-N. Pavlidou, "Innovative Chip Waveforms in Microcelluar DS/CDMA Packet Mobile Radio," IEEE Transactions on Communications, vol. 44, pp. 1413-1416, November 1996.

[18] CWTS-SWG2, LAS-CDMA, Physical Layer Aspects of TD-LAS High Speed Packet Technology, July 2001 